\title{
Harvesting energy from the vibration of a passing train using a single-degree-of-freedom oscillator
}

\author{
G. Gatti ${ }^{\text {a,* }}$, M.J. Brennan ${ }^{\text {b }}$, M.G. Tehrani ${ }^{\text {c }}$, D.J. Thompson ${ }^{\mathrm{c}}$ \\ a Department of Mechanical, Energy and Management Engineering, University of Calabria, Arcavacata di Rende (CS) 87036, Italy \\ ${ }^{\mathrm{b}}$ Departamento de Engenharia Mecãnica, Universidade Estadual Paulista (UNESP), Ilha Solteira, 15385-000 São Paulo, Brazil \\ ${ }^{\mathrm{c}}$ Institute of Sound and Vibration Research, University of Southampton, Southampton SO17 1BJ, United Kingdom
}

\section{A R T I C L E I N F O}

\section{Article history:}

Received 12 March 2015

Received in revised form

13 May 2015

Accepted 28 June 2015

Available online 15 July 2015

Keywords:

Energy harvesting

Train-induced vibration

Time-limited excitation

Transient vibration

\begin{abstract}
A B S T R A C T
With the advent of wireless sensors, there has been an increasing amount of research in the area of energy harvesting, particularly from vibration, to power these devices. An interesting application is the possibility of harvesting energy from the track-side vibration due to a passing train, as this energy could be used to power remote sensors mounted on the track for strutural health monitoring, for example. This paper describes a fundamental study to determine how much energy could be harvested from a passing train. Using a time history of vertical vibration measured on a sleeper, the optimum mechanical parameters of a linear energy harvesting device are determined. Numerical and analytical investigations are both carried out. It is found that the optimum amount of energy harvested per unit mass is proportional to the product of the square of the input acceleration amplitude and the square of the input duration. For the specific case studied, it was found that the maximum energy that could be harvested per unit mass of the oscillator is about $0.25 \mathrm{~J} / \mathrm{kg}$ at a frequency of about $17 \mathrm{~Hz}$. The damping ratio for the optimum harvester was found to be about 0.0045 , and the corresponding amplitude of the relative displacement of the mass is approximately $5 \mathrm{~mm}$.
\end{abstract}

(c) 2015 Elsevier Ltd. All rights reserved.

\section{Introduction}

Energy harvesting devices used to scavenge energy from ambient vibrations have been intensively studied during the past few years, mainly because of the need to power wireless devices, or to have autonomous solutions to augment the use of batteries [1,2]. A linear spring-mass-damper system has been the most common type of energy harvesting device, due to its simplicity. For harmonic excitation, good performance is achieved when the device is tuned so that its natural frequency coincides with the frequency of excitation [3].

Most of the previous studies in energy harvesting from vibrations have focussed on situations where the energy is harvested for a period of time long enough such that the initial transient response of the oscillator is negligible compared to the steady-state response [4-8]. If the damping in the harvester is low, however, which may be the case to increase the energy harvested in each cycle, the result would be that a relatively long initial transient would occur before the system

\footnotetext{
* Corresponding author. Tel.: + 390984 494157; fax: + 390984494673.

E-mail addresses: gianluca.gatti@unical.it (G. Gatti), mjbrennan0@btinternet.com (M.J. Brennan), m.ghandchi-tehrani@soton.ac.uk (M.G. Tehrani), djt@isvr.soton.ac.uk (D.J. Thompson).
} 
reaches its steady-state. This is a major limitation in some practical cases where there is time-limited excitation [9]. The initial transient may have a detrimental effect on the harvester performance if a classical design approach based on steadystate behaviour is considered.

In this paper, the energy harvested from the track-side vibration induced by the passage of a train is studied, as a specific practical application of time-limited excitation, where there is only significant vibration input for the duration of the train passage. A preliminary but not comprehensive investigation on this topic was recently presented by the authors in [10]. Such energy could be used to power or recharge batteries of rail-side equipment, such as wireless sensors for monitoring railway track health and temperature, warning light systems etc. Such applications are of particular importance in remote areas, where there is a lack of electrical infrastructure. A related area is the harvesting of energy from train suspensions [11], but this is considered to be a more classic case of harvesting energy from steady-state vibration and is well-covered in the literature.

Some research work in the area of scavenging energy from trains has recently focused on the design of specific harvesting devices from the technological and electro-mechanical point of view. For instance, an electromagnetic mechanism converting pulse-like linear vibration into regulated rotational motion was presented in [12]; a wide-band piezoelectric harvester was designed in [13] to generate power in various frequency regions; a piezoelectric generator installed under the sleeper, was used to scavenge energy from vertical vibrations of the track [14]; a device mounted on railties (sleepers) was used to converted the vertical displacement of the rail into electrical energy through mechanical amplification and rectification [15]; a comparison between an inductive coil device driven by the vertical rail displacement, and a piezoelectric device driven by the longitudinal strain produced by rail bending was presented in [16]. Several other power harvesting devices capable of scavenging power from the vertical deflection of railroad track are discussed in [17], and simulations on the maximum power potential for different prototypes along with their optimal number and location are presented in [18]. However, in all work cited above, no insight is given regarding the fundamental theory of vibration energy harvesting from time-limited excitation, which is the basis for the development of physical prototypes and devices.

The aim of this paper is thus to present a fundamental investigation on the maximum available energy that could be potentially harvested from a passing train using a linear single-degree-of-freedom oscillator. Using an acceleration time history of vertical vibration measured on a sleeper during the passage of an Inter-city 125 train in the United Kingdom, passing at a speed of about $195 \mathrm{~km} / \mathrm{h}$, the optimum mechanical parameters of a linear energy harvesting device are determined. Since the operational frequency range of the harvester is below $35 \mathrm{~Hz}$, the dynamics of the railway track are ignored. This would not be the case, however, when the train passes over a bridge, for example, where bridge dynamics could be exploited as well [19].

A preliminary numerical simulation is first performed to determine the optimum mechanical parameters. A theoretical study is then presented in which an approximate analytical formulation is proposed taking into consideration the optimal design of a linear single degree-of-freedom oscillator to scavenge energy from time-limited harmonic oscillations. The analytical study facilitates some general guidelines for the mechanical design of a harvester for train-induced vibrations.

\section{Optimal parameters of an energy harvester - numerical study}

The vertical acceleration of a sleeper was measured at Steventon on the Great Western Main Line in the UK as an Intercity 125 train at a speed of $195 \mathrm{~km} / \mathrm{h}(54.2 \mathrm{~m} / \mathrm{s})$ passed by. The track consists of continuously welded rail attached by spring clips and supported via rubber pads on concrete monoblock sleepers in ballast. The accelerometer was located at the center of the sleeper, but it may be noted that in the low frequency range below $50 \mathrm{~Hz}$ the whole sleeper vibrates as a rigid body. The track is raised on a shallow embankment, approximately $0.7 \mathrm{~m}$ above the level of the surrounding fields. The ground at the site consists of deep layers of clay; for more details see [20]. The train consists of two diesel power cars, one at each end, with seven passenger coaches between them. Each vehicle is supported by four wheel-sets arranged in two bogies, each of which has a wheelbase of $2.6 \mathrm{~m}$. The power cars are about $18 \mathrm{~m}$ long, while the passenger coaches are about $23 \mathrm{~m}$ long. Seven seconds of data were recorded at a sampling frequency of $1 \mathrm{kHz}$ as the train passed by, and the resulting acceleration signal is shown in Fig. 1(a). The power spectral density of the acceleration signal is shown in Fig. 1(b) up to a frequency of $35 \mathrm{~Hz}$. At the top of this figure, another frequency axis is shown, which is the actual frequency normalized by $S / L$, where $S$ is the train speed and $L$ is the length of the passenger carriages. It can be seen that the peaks in the spectrum appear to be integer numbers of the ratio $S / L$, which were referred to as the trainload dominant frequencies in [21]. It can also be seen that the maximum acceleration occurs when the non-dimensional frequency is equal to 7 , which corresponds to a frequency of about $17 \mathrm{~Hz}$ for this speed.

The acceleration signal shown in Fig. 1(a) is considered to be the base input to the energy harvesting device shown in Fig. 2, which is a single-degree-of-freedom mass-spring-damper system encased in a rigid housing. It is assumed that the vibration of the energy harvesting device does not affect the vibration of the sleeper. For the sake of simplicity in this fundamental study, it is further assumed that the energy harvested is the same as the energy dissipated by the damper, and this identifies the ideal upper-limit case where no mechanical loss is considered. As the harvester is encased in a rigid housing, the maximum relative displacement of the mass is limited to $|z|_{\max }$, where $z=x-y$ is the relative displacement between that of the mass $x$, and the casing $y$. The equation of motion for the energy harvesting device is given by

$m \ddot{z}+c \dot{z}+k z=-m \ddot{y}$ 

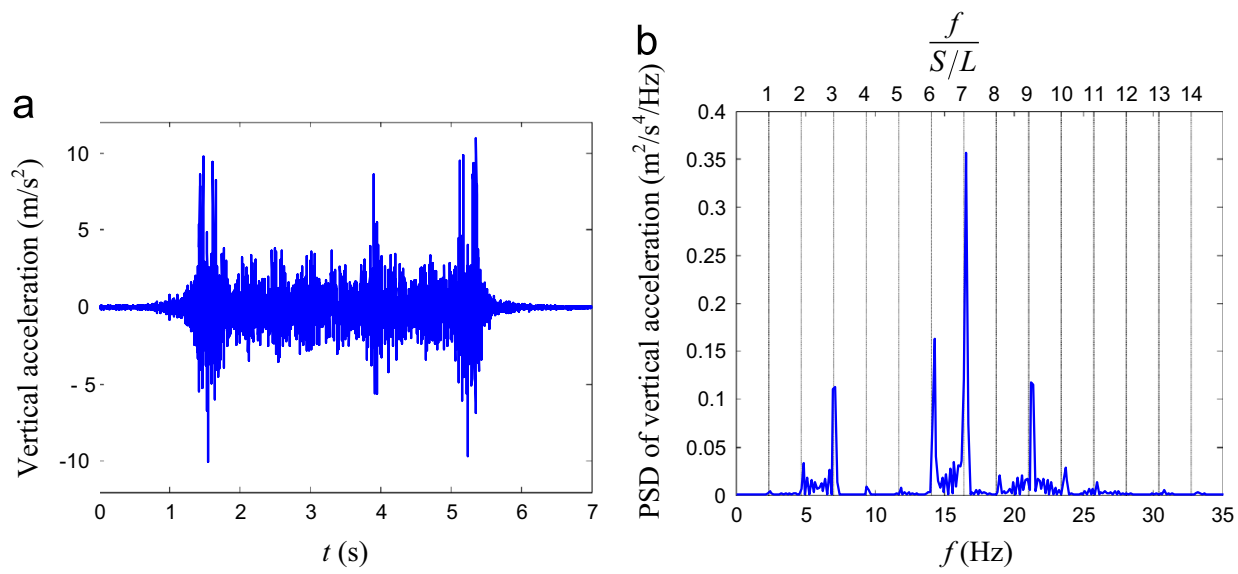

Fig. 1. Measured vertical acceleration of a sleeper as an Inter-city 125 train passes by: (a) time history and (b) power spectral density (PSD). $S / L$ is the ratio of the speed of the train to the length of a carriage.

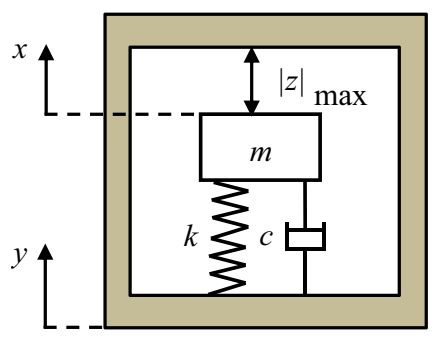

Fig. 2. Schematic of the energy harvester. The energy dissipated by the damper is considered to be the energy available for harvesting.

where $m, k$ and $c$ are mass, stiffness and damping respectively, and the over dot denotes differentiation with respect to time. The energy harvested at time $t_{\mathrm{e}}$ is given by

$$
E\left(t_{\mathrm{e}}\right)=\int_{0}^{t_{\mathrm{e}}} c \dot{z}^{2} \mathrm{~d} t
$$

From Fig. 1 it can be seen that the train induces vibrations from approximately $1.5 \mathrm{~s}$ to about $5.5 \mathrm{~s}$, so that the input duration can be considered to be approximately $4 \mathrm{~s}$. During this period the energy harvester undergoes forced vibration, and when the train has passed it experiences free vibration. Only energy harvested during the forced vibration phase is considered in this paper.

To determine the best natural frequency $\omega_{0}=\sqrt{\mathrm{k} / \mathrm{m}}$, and damping ratio $\zeta=c /(2 \sqrt{\mathrm{km}})$, for the energy harvester subject to the base acceleration time history in Fig. 1(a), a numerical simulation is carried out. The mass is set to $1 \mathrm{~kg}$ and the natural frequency is varied from $0.1 \mathrm{~Hz}$ to $35 \mathrm{~Hz}$ with an increment of $0.07 \mathrm{~Hz}$. For each natural frequency the damping is set to a value in the range $1 \times 10^{-4}<\zeta<5 \times 10^{-2}$. Eq. (1) is first integrated numerically to determine the relative velocity using the acceleration of the sleeper as the input to the base. The relative velocity is then substituted into Eq. (2) with $t_{\mathrm{e}}$ set to $5.5 \mathrm{~s}$, to calculate the energy harvested. The simulation for each natural frequency is repeated until the energy harvested is maximized.

The results are shown in Fig. 3 as a continuous solid blue line (the red circles in the figure are discussed in Section 3). Fig. 3(a) shows the maximum energy that can be harvested for each natural frequency, and for a mass of $1 \mathrm{~kg}$. Fig. 3(b) shows the corresponding damping ratio, which is optimal for each natural frequency and Fig. 3(c) shows the maximum value of the relative displacement that occurs during the whole simulation. From Fig. 3(a), it can be seen that the energy peaks correspond to the five highest peaks in the acceleration spectrum, which correspond to trainload dominant frequencies. The maximum energy harvested occurs when the acceleration is a maximum. From Fig. 3(b) it is observed that the optimum damping ratio varies considerably. It can also be observed that the optimum damping ratio is relatively small for natural frequencies corresponding to the peaks in Fig. 3(a). As expected, the relative displacement is larger when the energy harvested is larger, which can be seen in Fig. 3(c). It can also be seen that the largest relative displacement does not occur for the largest amount of energy harvested. The conclusion form the numerical optimization is that the harvester should be tuned to a natural frequency of about $17 \mathrm{~Hz}$ and have a damping ratio of about 0.0045 to maximize the energy harvested. This results in a maximum relative displacement of about $5 \mathrm{~mm}$. Such a device will produce about $0.25 \mathrm{~J} / \mathrm{kg}$ of oscillator mass as the train passes. 

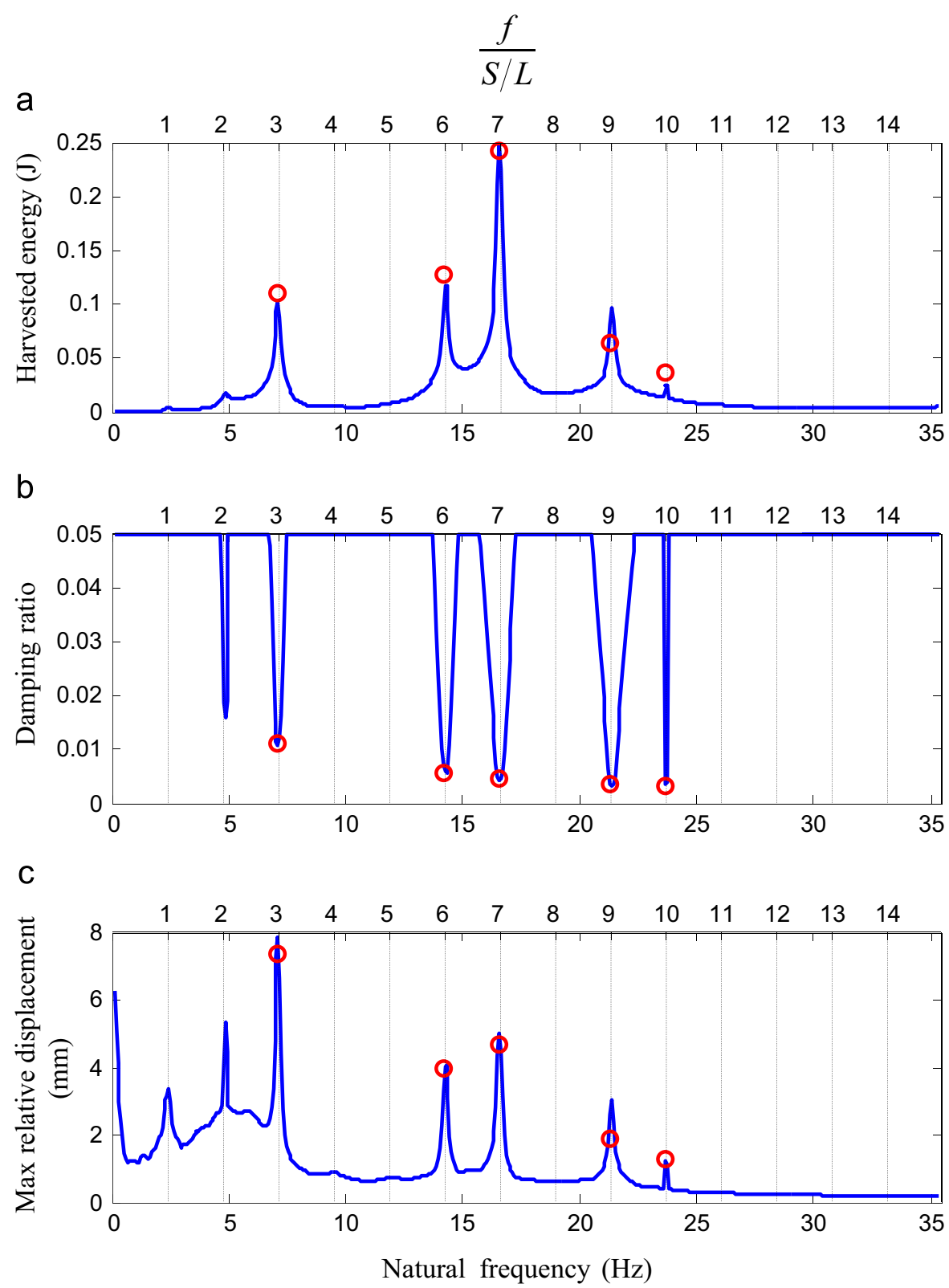

Fig. 3. Results for an energy harvester for each natural frequency. Numerical optimization (blue solid line). Approximate analytical expression (red circles). (a) Maximum energy harvested, (b) corresponding optimum damping, and (c) resulting maximum displacement. (For interpretation of the references to color in this figure legend, the reader is referred to the web version of this article.)

\section{Optimal parameters of an energy harvester - analytical study}

To see more clearly the features of the input vibration to the energy harvester, the velocity is calculated from the acceleration signal in Fig. 1(a) by computing the cumulative integral via the trapezoidal method. This is shown in Fig. 4(a), from which it can be seen that the vibration appears to be predominantly time-periodic, except at the start and end of the train passage. The larger vibration at the ends is due to the heavier power cars. Using the time history corresponding to the time period highlighted as a red solid line in Fig. 4(a), the Fourier coefficients of the input velocity are determined. The first 15 of these coefficients are shown in Fig. 4(b). The single frequency vibration corresponding to these coefficients can then be used as a base input into the energy harvester.

The relative displacement of the mass in the energy harvester, due to single frequency displacement excitation of the base given by $y(t)=Y \sin (\omega t)$ that starts from rest at $t=t_{0}=0$ and finishes at $t=t_{\mathrm{e}}$, may be obtained by the solution 
a

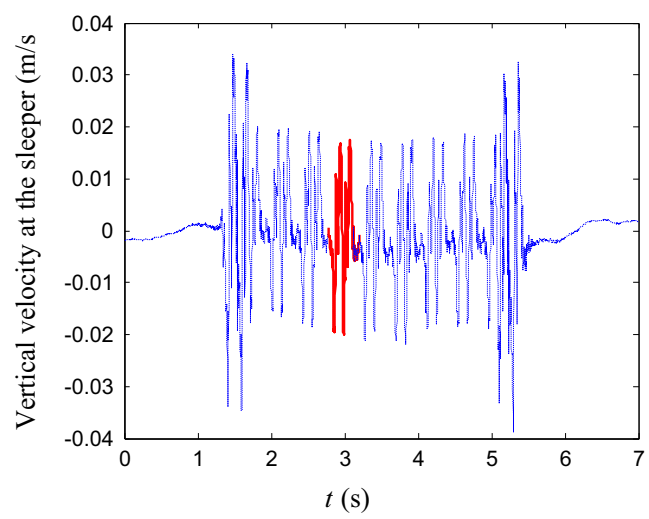

b $\frac{f}{S / L}$

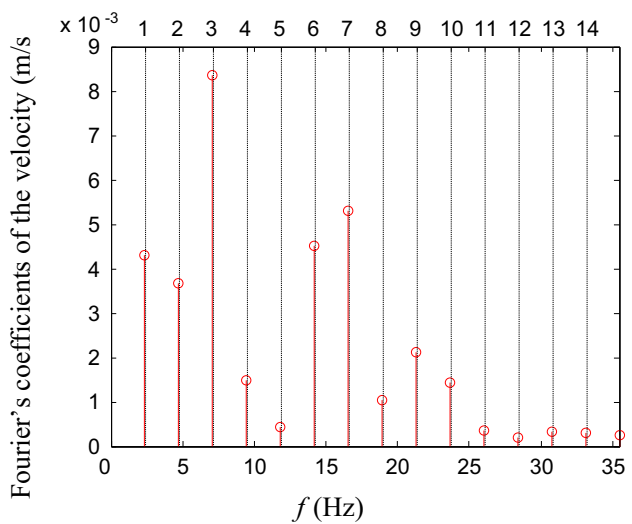

Fig. 4. Vertical velocity of a sleeper as an Inter-city 125 train passes by: (a) time history calculated from the acceleration time history (blue dotted line) and periodic sample used to calculate the Fourier coefficients (red solid line) and (b) Fourier coefficients corresponding to the red solid line in (a). (For interpretation of the references to color in this figure legend, the reader is referred to the web version of this article.)

to Eq. (1) as

$$
z(t)=\left|\frac{Y \omega^{2}}{\omega_{0}^{2}-\omega^{2}+2 j \zeta \omega \omega_{0}}\right|\left(\sin (\omega t+\phi)-\frac{\omega}{\omega_{\mathrm{d}}} \mathrm{e}^{-\zeta \omega_{0} t} \sin \left(\omega_{\mathrm{d}} t+\alpha\right)\right) \quad \text { for } \quad 0 \leq t \leq t_{\mathrm{e}},
$$

where

$$
\phi=-\operatorname{atan}\left(\frac{2 \zeta \omega \omega_{0}}{\omega_{0}^{2}-\omega^{2}}\right), \alpha=-\operatorname{atan}\left(\frac{2 \omega_{0}^{2} \zeta \sqrt{1-\zeta^{2}}}{\omega_{0}^{2}-\omega^{2}-2 \omega_{0}^{2} \zeta^{2}}\right), \omega_{d}=\omega_{0} \sqrt{1-\zeta^{2}} \text {, and } j=\sqrt{-1}
$$

Now, the maximum energy that can be harvested from an oscillator for steady-state excitation occurs when the natural frequency is tuned to the excitation frequency. If this tuning condition is applied and it is assumed that damping is light such that $\zeta^{2}<<1$, then the relative displacement given by Eq. (3) becomes

$$
z(t) \approx \frac{Y}{2 \zeta}\left(1-\mathrm{e}^{-\zeta \omega_{0} t}\right) \sin \left(\omega_{0} t\right)
$$

An illustration of the behaviour of the energy harvester subject to time-limited harmonic base excitation is shown in Fig. 5. There could be up to three distinct phases; during excitation of the base there is a transient response, which may be followed by a steady-state response, and after the excitation has finished, the vibration of the harvester mass decays away freely. To determine the energy harvested, Eq. (4) is substituted into Eq. (2). Noting that $t_{\mathrm{e}}=2 \pi n / \omega_{0}$, the energy harvested at time $t_{\mathrm{e}}$ after $n$ cycles of excitation, normalized by the kinetic energy of the oscillator mass excited with the same velocity as the base, is found to be

$$
\hat{E}(n)=\frac{E(n)}{\frac{1}{2} m \dot{Y}^{2}} \approx\left(\frac{\pi n}{\zeta}\right)-\left[\frac{3+\mathrm{e}^{-4 \pi n \zeta}-4 \mathrm{e}^{-2 \pi n \zeta}}{4 \zeta^{2}}\right]
$$

where $\dot{Y}$ is the amplitude of the base velocity. To determine the physical significance of Eq. (5) it is necessary to study the various terms in the equation, also considering the vibration response shown in Fig. 5. Examining the time history of the response of the suspended mass of the oscillator, it can be seen that it takes some time for the vibration level to reach the steady-state response, which starts at $t_{\mathrm{s}}$. If the system was at steady-state for $n$ cycles, the normalized energy harvested during this phase would be $\hat{E}(n)=(\pi n / \zeta)$, which can simply be determined from calculating the energy harvested in one cycle and multiplying by the number of cycles. Note that this is the term in the round brackets on the right-hand side of Eq. (5). Note also that in this case the amount of energy harvested is inversely proportional to the amount of damping in the system. However, damping cannot be reduced to an arbitrarily small value, because as damping is reduced the relative displacement between the oscillator mass and the casing increases. As the system is excited at its resonance frequency, the relationship between the minimum damping and the maximum relative displacement is given by $\zeta_{\min }=Y /\left(2 Z_{\max }\right)$, where $Z_{\text {max }}$ is the maximum amplitude of the relative displacement.

The term in the square brackets in Eq. (5) is related to the time it takes for the vibration of the mass to reach steady-state. Note that as $\pi n \zeta$ tends to infinity, this term tends to zero, which simply means that if $n$ is sufficiently large, then the energy harvester will be in the steady-state for most of the time. Thus, if $n$ is fixed, it is desirable for $\zeta$ to be large. It can be seen, therefore, that there are two competing factors in the determination of the best value of $\zeta$; to increase the energy harvested due to steady-state vibration a small value of $\zeta$ is required, and to minimize the time that it takes to reach steady-state 

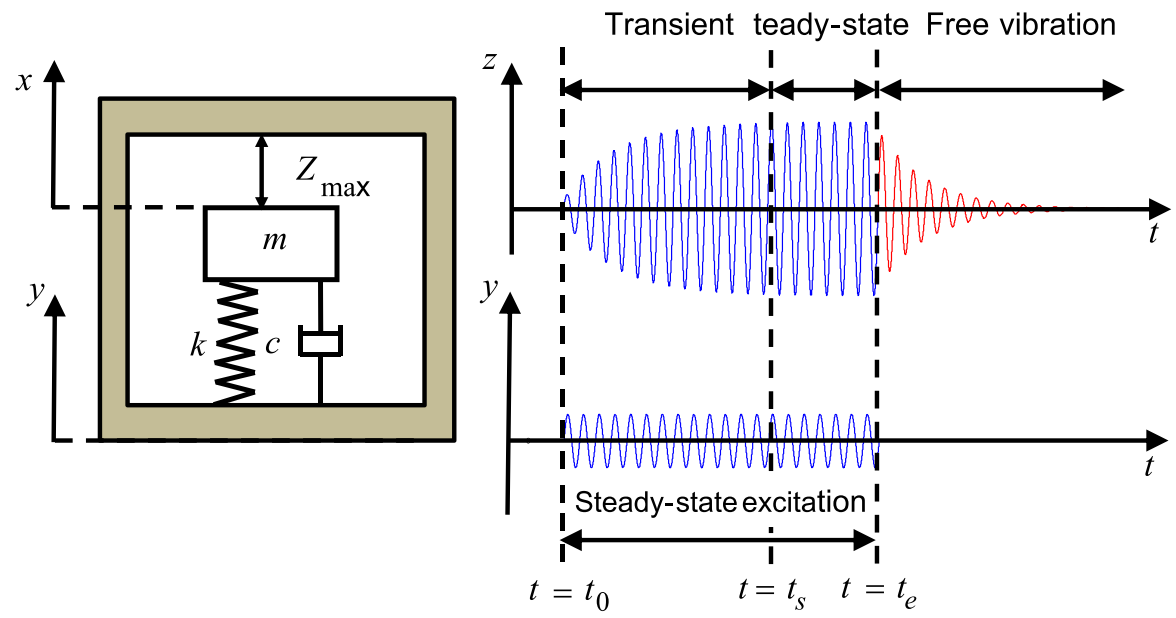

Fig. 5. Energy harvester subject to time-limited harmonic base excitation.

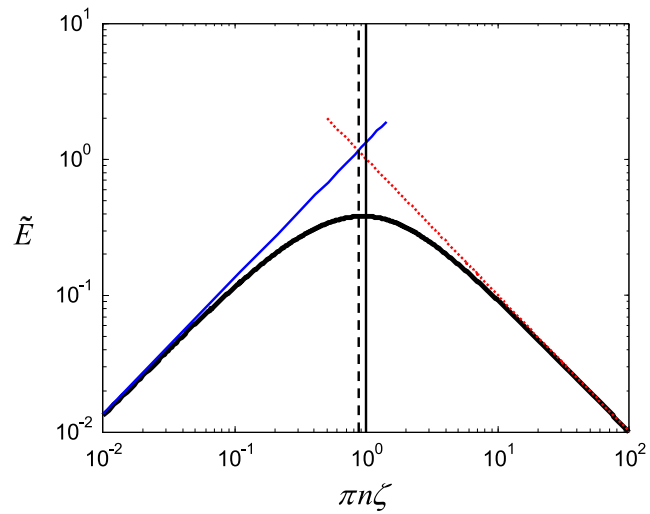

Fig. 6. Non-dimensional energy as a function of $\pi n \zeta$ as thick solid black line. Thin solid blue line is the asymptote given by $\tilde{E}=4 \pi n \zeta / 3$; thin dotted red line is the asymptote given by $\tilde{E} \approx 1 /(\pi n \zeta)$. The vertical solid line is for $\pi n \zeta=1$; the vertical dashed line is for $\pi n \zeta=\sqrt{3} / 2 \approx 0.866$. (For interpretation of the references to color in this figure legend, the reader is referred to the web version of this article.)

excitation a large value of $\zeta$ is required. Rather than plotting Eq. (5), it is preferable to manipulate this equation to make $\pi n \zeta$ the independent variable. In this case it becomes

$$
\tilde{E}(\pi n \zeta)=\frac{\hat{E}(n)}{(\pi n)^{2}}=\left(\frac{1}{\pi n \zeta}\right)-\left[\frac{3+\mathrm{e}^{-4 \pi n \zeta}-4 \mathrm{e}^{-2 \pi n \zeta}}{(2 \pi n \zeta)^{2}}\right]
$$

This is plotted in Fig. 6 which shows the importance of $\pi n \zeta$ as the key group of parameters when choosing the optimum damping to maximize the energy harvested for a particular device. The reason for plotting the graph on a $\log -\log$ scale, is that it is clear that there are two asymptotes for $\pi n \zeta<<1$ and for $\pi n \zeta>>1$, which, for a given level of damping, correspond to a system where there is a small and large number of cycles respectively. When $\pi n \zeta<<1$, Eq. (6) becomes $\tilde{E}=4 \pi n \zeta / 3$, and for $\pi n \zeta>>1$, Eq. (6) becomes, $\tilde{E} \approx 1 /(\pi n \zeta)$. It is not possible to determine a closed-form expression for the maximum of Eq. (6), which is evident in Fig. 6, because of the exponentials due to the transient. However, an approximate expression can be determined by setting the two asymptotes to be equal, which is when $\pi n \zeta=\sqrt{3} / 2 \approx 0.866$. This should be compared with the optimum value calculated by equating to zero the derivative of Eq. (6) with respect to $\pi n \zeta$, and solving numerically to give $\pi n \zeta=0.9463$. Examining Fig. 6 , it can be seen that the maximum energy harvested is not particularly sensitive to $\pi n \zeta$ when it is close to $\pi n \zeta=1$. Thus it is suggested that a simple rule to determine the optimum damping is given by

$$
\zeta_{\text {opt }}=\frac{1}{\pi n}
$$

Eq. (7) indicates that in order to maximize the harvested energy, the optimum damping should be inversely proportional to the input duration, expressed in terms of excitation cycles. It is also interesting to note that if the optimum damping determined from Eq. (7) is substituted into Eq. (4), which gives the expression for the relative displacement of the harvester mass, the corresponding displacement amplitude is about $86 \%$ of the steady-state amplitude when $t=t_{\mathrm{e}}$, i.e. at the end of the forced excitation, after which the vibration of the harvester mass decays away freely. Thus, for a given input duration, i.e. for a given value of $n$, the optimum energy is harvested if the oscillator never reaches steady-state during the excitation 
a
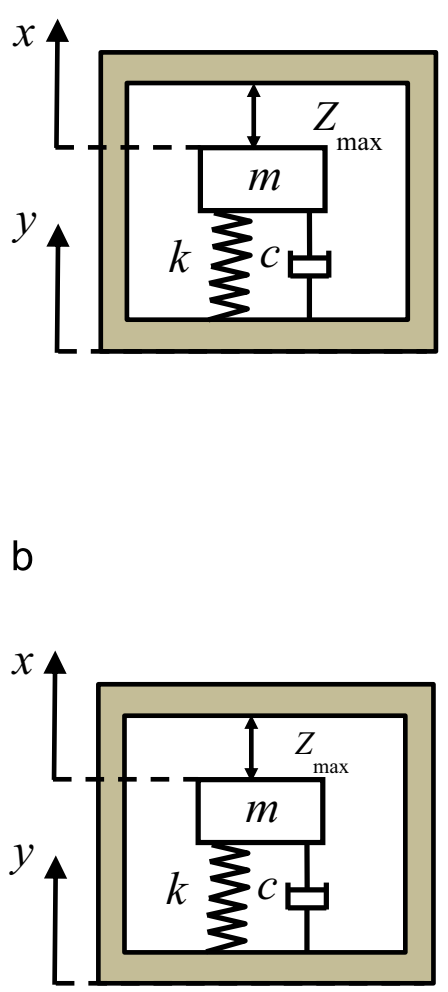

b
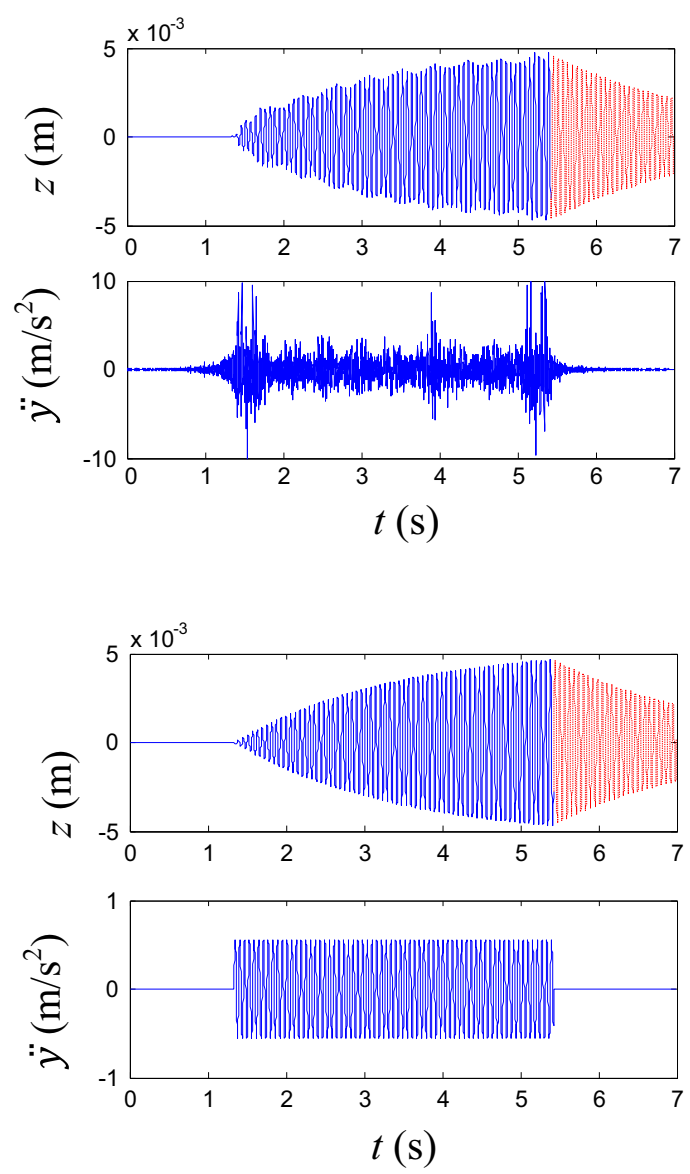

Fig. 7. Behaviour of the optimized energy harvester in the time-domain: (a) when subject to train-induced vibration and (b) when subject to its harmonic component at about $17 \mathrm{~Hz}$.

phase. Furthermore, combining Eqs. (5) and (7), the normalized optimum energy harvested for $n$ cycles of vibration is found to be

$$
\hat{E}(n) \approx(\pi n)^{2}\left(\frac{1-\mathrm{e}^{-4}+4 \mathrm{e}^{-2}}{4}\right) \approx 0.38(\pi n)^{2}
$$

which, in dimensional form, may be written in terms of the input duration as

$$
\frac{E\left(t_{\mathrm{e}}\right)}{m} \approx 0.0475 \ddot{Y}^{2} t_{\mathrm{e}}^{2}
$$

from which it is evident that the harvested energy is proportional to the square of the input duration and to the square of the amplitude of the base acceleration, $\ddot{Y}$, as was noted by observing the results of the numerical optimization in the previous section.

The simple approximate expression given in Eq. (9) is applied to the measured data from the train-induced vibrations described in Section 2. In particular, the frequencies corresponding to the five largest peaks in Fig. 3(a) are multiplied, respectively, by the corresponding Fourier coefficients of the input velocity in Fig. 4(b) to obtain the amplitude of the input acceleration. The square of these is then multiplied by the square of the input duration, $4 \mathrm{~s}$, and the resulting energy is plotted by red circles in Fig. 3(a). Corresponding results for the approximate optimum damping ratios are determined by applying Eq. (7) and are plotted in Fig. 3(b). Likewise the approximate relative displacement amplitudes are calculated using Eq. (4) and are plotted in Fig. 3(c). It can be observed that there is good agreement between the numerical results and the approximate analytical results.

As a final verification, Fig. 7(a) and (b) shows the results of the time-domain simulations performed on the optimized harvester, when subject to the base excitation measured at the sleeper which is shown in Fig. 1(a), and when subject to its harmonic component at about $17 \mathrm{~Hz}$, which is obtained by multiplying the corresponding velocity Fourier coefficient in Fig. 4(b) by its frequency, respectively. It can be observed that the responses are very similar in both cases for the optimized harvester. This shows that the analytical formulation based on the assumption of a single harmonic excitation, can be 
effectively adopted to describe the actual behaviour in the case of an excitation induced by a train passage, which is observed to be very different from single harmonic excitation. In particular, for the case of a single-degree-of-freedom harvester, the behaviour is dominated by the response to the frequency component of the excitation input, which corresponds to the natural frequency. Furthermore, it can be observed from Fig. 7 that the amplitude of the relative displacement of the harvester mass does not reach steady-state by the end of the input duration, after which it decays away freely, as discussed earlier.

\section{Conclusions}

This paper has described a fundamental investigation into how much energy can be harvested from the vertical vibration of the track due to a passing train using a single linear energy harvesting device. Despite the preliminary nature of this study, it does indicate how much energy can be potentially harvested from a typical train pass by. It was found that the harvester should be tuned to a frequency at which the vertical acceleration is the greatest, and this corresponds to one of the train-load frequencies. It was also found that the transient part of the forced vibration is very important and that the damping should be chosen such that the vibration of the mass of the energy harvester does not reach steady-state during the passage of the train.

Approximate analytical expressions have been derived and a simple strategy has been proposed to optimize the mechanical parameters of the harvesting devices. In particular, expressions for the optimum damping and corresponding maximum energy have been determined. It has been found that the optimum harvested energy per unit mass is proportional to the product of the square of the input acceleration amplitude and the square of the input duration. An expression has also been determined which gives the maximum relative displacement amplitude achieved by the oscillator mass, which facilitates the dimensioning of the harvester casing.

For the specific case studied it was found that the maximum energy that could be harvested is about $0.25 \mathrm{~J} / \mathrm{kg}$ of energy harvester mass at a frequency of about $17 \mathrm{~Hz}$. The damping ratio for the harvester was found to be about 0.0045 , and the corresponding amplitude of the relative mass displacement is approximately $5 \mathrm{~mm}$.

\section{References}

[1] S. Roundy, P.K. Wright, J. Rabaey, A study of low level vibrations as a power source for wireless sensor nodes, J. Comput. Commun. 26 (2002) $1131-1144$.

[2] C. Knight, J. Davidson, S. Behrens, Energy options for wireless sensor nodes, Sensors 8 (2008) 8037-8066.

[3] N.G. Stephen, On energy harvesting from ambient vibration, J. Sound Vib. 293 (2006) 409-425.

[4] E. Lefeuvre, A. Badel, C. Richard, L. Petit, D. Guyomar, A comparison between several vibration-powered piezoelectric generators for standalone systems, Sens. Actuators A: Phys. 126 (2006) 405-416.

[5] M. Lallart, D. Guyomar, Y. Jayet, L. Petit, E. Lefeuvre, T. Monnier, P. Guy, C. Richard, Synchronized switch harvesting applied to selfpowered smart systems: piezoactive microgenerators for autonomous wireless receiver, Sens. Actuators A: Phys. 147 (2008) $263-272$.

[6] L. Garbuio, M. Lallart, D. Guyomar, C. Richard, D. Audigier, Mechanical energy harvester with ultralow threshold rectification based on SSHI nonlinear technique, IEEE Trans. Ind. Electron. 56 (2009) 1048-1056.

[7] S.R. Anton, H.A. Sodano, A review of power harvesting using piezoelectric materials (2003-2006), Smart Mater. Struct. 16 (2007) R1-R21.

[8] H.A. Sodano, D.J. Inman, G. Park, A review of power harvesting from vibration using piezoelectric materials, Shock Vib. Dig. 36 (2004) 197-205.

[9] M. Lallart, D.J. Inman, D. Guyomar, Transient Performance of energy harvesting strategies under constant force magnitude excitation, J. Intell. Mater. Syst. Struct. 21 (2010) 1279-1291.

[10] G. Gatti, M.J. Brennan, M.G. Tehrani, D.J. Thompson, On energy harvesting form time-limited excitations: simulations and case study, in: Proceedings of ISMA 2014 - International Conference on Noise and Vibration Engineering and USD 2014 - International Conference on Uncertainty in Structural Dynamics, Leuven, Belgium, September 2014, pp. 981-991.

[11] T. Guo, R. Kerley, D.S. Ha, Development of a power conditioning circuit for railcar energy harvesting, in: Proceedings of the IEEE 56th International Midwest Symposium on Circuits and Systems, MWSCAS, Columbus (OH), United States, August 2013, pp. 513-516.

[12] J.J. Wang, G.P. Penamalli, L. Zuo, Electromagnetic energy harvesting from train induced railway track vibrations, in: Proceedings of the 8th IEEE/ASME International Conference on Mechatronic and Embedded Systems and Applications, MESA, Suzhou, China, July 2012, pp. 29-34.

[13] J. Li, S. Jang, J. Tang, Implementation of a piezoelectric energy harvester in railway health monitoring, in: Proceedings of SPIE - The Internationa Society for Optical Engineering - Sensors and Smart Structures Technologies for Civil, Mechanical, and Aerospace Systems, San Diego (CA), United States, March 2014, article number 90612Q.

[14] Y. Tianchen, Y. Jian, S. Ruigang, L. Xiaowei, Vibration energy harvesting system for railroad safety based on running vehicles, Smart Mater. Struct. 23 (2014) 125046.

[15] S.E. Hansen, A. Pourghodrat, C.A. Nelson, M. Fateh, On-track testing of a power harvesting device for railroad track health monitoring, in: Proceedings of SPIE - The International Society for Optical Engineering - Health Monitoring of Structural and Biological Systems, San Diego (CA), United States, March 2010, article number 76500Y.

[16] C.A. Nelson, S.R. Platt, D. Albrecht, V. Kamarajugadda, M. Fateh, Power harvesting for railroad track health monitoring using piezoelectric and inductive devices, in: Proceedings of SPIE - The International Society for Optical Engineering - Active and Passive Smart Structures and Integrated Systems, San Diego (CA), United States, March 2008, article number 69280R.

[17] A. Pourghodrat, C.A. Nelson, S.E. Hansen, V. Kamarajugadda, S.R. Platt, Power harvesting systems design for railroad safety, Proc. Inst. Mech. Eng F: J Rail Rapid Transit 228 (2014) 504-521.

[18] K.J. Phillips, C.A. Nelson, M. Fateh, Simulation and control system of a power harvesting device for railroad track health monitoring, in: Proceedings of SPIE - The International Society for Optical Engineering - Health Monitoring of Structural and Biological Systems, San Diego (CA), United States, March 2011, article number 79840D.

[19] P. Cahill, N.A.N. Nuallain, N. Jackson, A. Mathewson, R. Karoumi, V. Pakrashi, Energy harvesting from train-induced response in bridges, J. Bridge Eng. 19 (2014) 04014034

[20] N. Triepaischajonsak, D.J. Thompson, C.J.C. Jones, J. Ryue, J.A. Priest, Ground vibration from trains: experimental parameter characterization and validation of a numerical model, Proc. Inst. Mech. Eng F: J. Rail Rapid Transit 225 (2011) 140-153.

[21] S. Ju, H. Ling, J. Huang, Dominant frequencies of train-induced vibrations, J. Sound Vib. 319 (2009) $247-259$. 